\title{
Bio Metric based Challan System for Smart Local Transport in India
}

\author{
Ashwini Jewalikar \\ Asst Prof CE Dept PICT Pune
}

\author{
Tejal Jadhav
}

\begin{abstract}
Maintaining discipline in public transport system is need of today. since traffic on the roads is increasing day by day and so the pollution. Traffic signals plays very important role in maintaining discipline in public/local transportation systems in urban cities like Mumbai, Pune in India. Many underage children drives vehicles, and driving without license may cause accidents. Maintaining the discipline in public transport is core responsibility of each and every citizen. Due to corruption and non adherence to rules are major hurdle in maintaining the local transport discipline of India. Proposed, "Bio metric based Challan system for RTO" which makes use of human characteristics such as fingure prints and Eris to perform verification process against the RTO database stored under "licensed users", which will help to reduce the rate of corruption and make people think to maintain the discipline in local transport which may reduce the count of traffic rule brake cases.
\end{abstract}

\section{General Terms}

Internet of Things, Aurduino Uno, Raspberry Pi, Python

\section{Keywords}

IoT, Python

\section{INTRODUCTION}

RTO : The Regional Transport Office or Regional Transport Authority(RTO/RTA) is the organization of the Indian government responsible for maintaining a database of drivers and a database of vehicles for various states of India[5]. The RTO [5] issues driving licenses, organizes collection of vehicle excise duty(also known as road tax and road fund license) and sells personalized registrations. The RTO identifies non-taxed vehicles, and keeps track of cars moving in various Indian states, they also have set of rules and regulations to be followed during local transport such as maintaining speed limits, wearing helmet etc.

Now a days in India digitization of the things is in boom and almost every citizen's Aadhar card details are been linked to the bank account.This technical revolution helps in digitization and development of many applications for the sake of goodness of a common man.

Objective of RTO Based challan system is to let the users follow the RTO traffic rules during local transportation and reduce the corruption rate. Due to linking of Aadhar card details , the bank account \& RTO server, fine deduction due to breaking the RTO rules becomes easy and immediate and receipt is generated with notification on mobile of the person who has broken the rule. Apart from it, notification is sent to the user. This will make the people compulsory to maintain discipline during local transport and collect fine from those who don't follow traffic rules. It helps traffic policeman to keep record of the people who frequently disobeys the discipline and charge them accordingly.

\section{RELATED WORK}

An important and very reliable human identification method is fingerprint identification [1]. A system that consists of a smart card capable of storing the fingerprint information of person is proposed in [2], in this system, while issuing the license, the specific person's fingerprint is to be stored in the card. One of the limitation of it is vehicles such as cars, bikes etc. should have a card reader capable of reading the license and the same automobile should have the facility of fingerprint reader device. [2]. Driving license system is a very difficult task for the government to monitor[3] hence, all the citizens' images will be scanned and recorded. Whenever a citizen crosses the traffic rules, the police can scan his/her image and can collect penalty / fine from the defaulter. Using this method, the police can track the history of the driver such as rules broken before ,charged fine etc. Proposed bio metric based driving license monitoring system is very easy and convenient to monitor. Various methods for fingerprint matching scheme are available which are generally used for identification of a person. In Paper[4] author provided the various Minutiae and the Texture Features for identify the specific pattern are integrating the advantage of fingure print identification with the digital identities of a citizen (Adhar and RTO details) to reduce the traffic rule braking cases and corruption rate.

\section{PROPOSED SYSTEM}

Proposed system consists of interconnection of servers (RTO server, Adhar Server etc.) with a Raspberry pi mounted with bio metric sensor and Traffic Policeman's mobile phone, as shown in fig 1.

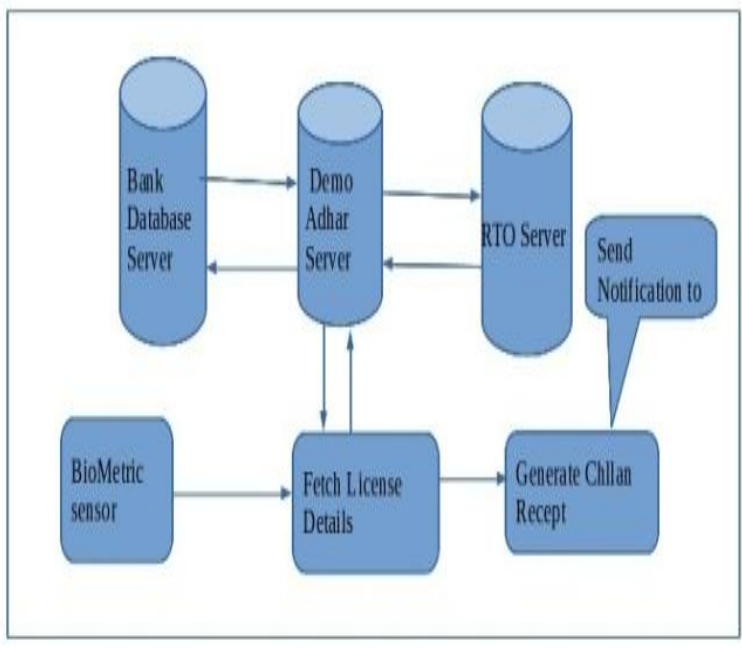

Fig:1 Proposed System architecture

\subsection{Proposed System Architecture}

In the proposed prototype model architecture, Bank database Server, Adhar Server and Local RTO server are connected 
together. Local Traffic policeman have a Mobile Smartphone connected to a Raspberry pi/Aurduino Uno device[6]which has a fingerprint sensor and a wifi module. This Raspberry pi/ Aurduino Uno[6][7] is connected to Adhar server.

\subsection{Algorithm}

1. Scan Fingerprint of user

2. Fetch the license no. and other information of user from RTO server.

3. Select rules broken (over speed, no helmet, no license etc.)

4. Deduct amount from user bank

5. Generate fine receipt

6. Notification send to user and admin(Police).

Users/Citizens are first get registered with the server application running on RTO server as soon as they receives driving license form RTO office.

This registration process includes collecting fingerprint samples, eris samples and ADHAR details and bank account details of the person who has issued driving license by RTO authority. All the information collected will be stored in a database of RTO server.

If a person breaks the traffic rule and caught by a traffic policeman, he/she will take his/her fingerprints for verification. These sample finger prints will be verified against the database stored on Adhar server. Aurduino Uno board connected with Bio metric sensor will connect to Adhar Server and the application running on mobile phone of the Policeman(connected to Aurduino Uno/Raspberry pi) will check whether the person has issued a valid license or not and how many times he/she has broken the traffic rules. after verification using bio metric characteristics, a list of charges against violation of traffic rules will be displayed on Policeman's mobile and he/she will get notified about the amount of fine needs to be paid . system will be connected with bank using Adhar-card. automatically fine is been deducted from that persons account, and notification will be sent for the same either on mobile or through email.

\subsection{Implementation, Experimental set up}

Hardware used: to collect fingerprints of the rule broker/citizen GT/511C3 is used which is to be connected to Aurduino Uno board. Wifi module is also interfaced with Aurduino for connecting to RTO server.

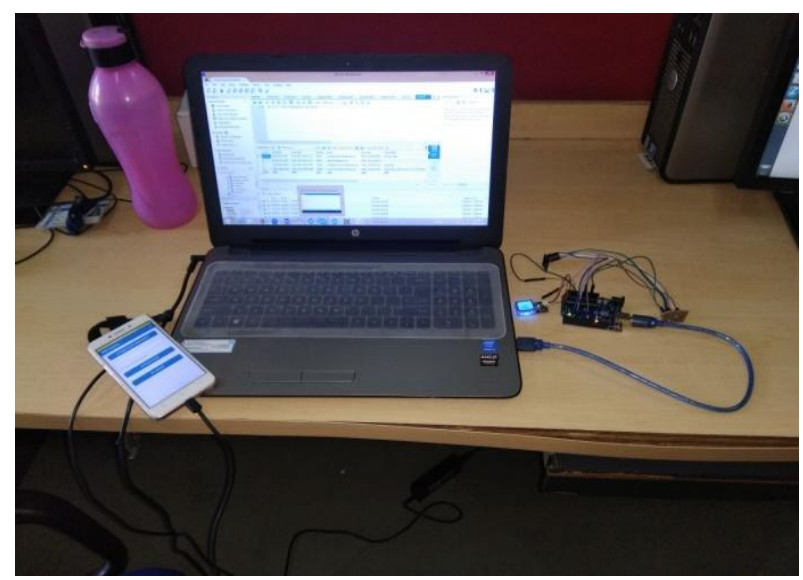

Fig.2 Test Bed

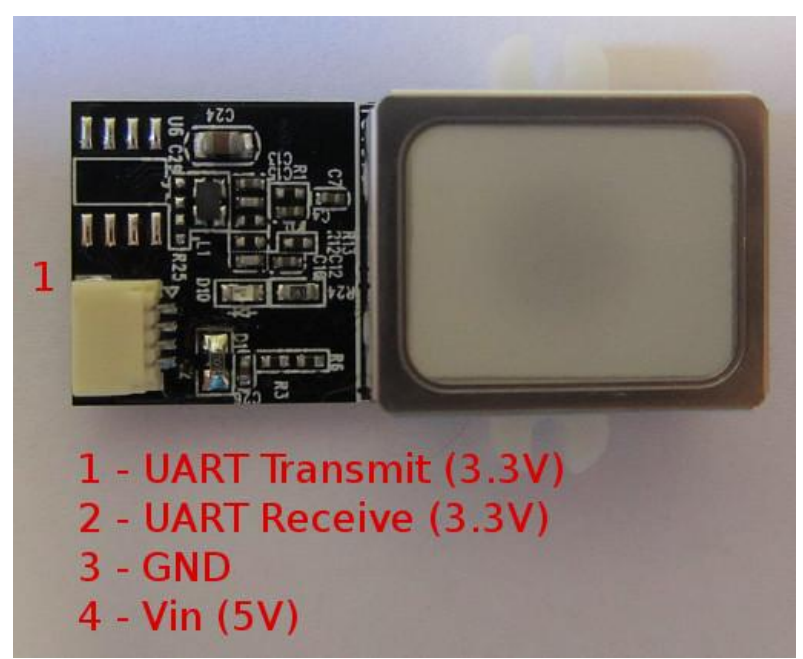

Fig.3: Biometric Sensor

Software Details:Traffic Policeman has to use an Android Application to choose from the list of rules set by RTO and accordingly fine/Challan will be charged .This Mobile phone is connected to RTO server,Adhar server via Aurduino Uno to fetch the details of the citizen,such as driving license Number,vehicle number, Name etc.

GUI for Traffic Policeman: The GUI for the application running on Traffic policeman's Mobile is shown in fig 4.

\section{RTO Licence}

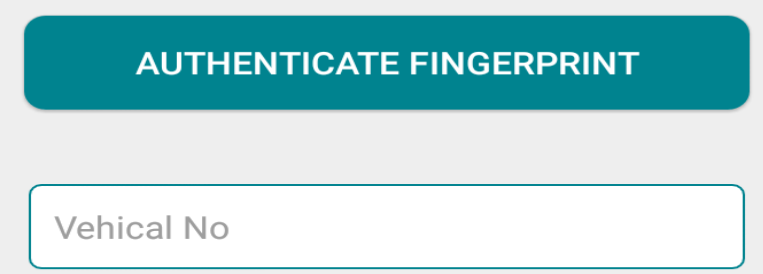

Use License number.

\section{AUTHENTICATE}

\section{MY REPORT}

\section{Fig.4:Application Running on Traffic Policeman's Mobile}

\section{CONCLUSION}

Proposed smart local transport system "Bio metric based Callahan system for RTO" which makes use of human characteristics such as finger prints and Eris to perform for verification process against the RTO database stored under "licensed users" will help to reduce the corruption and the count of traffic rule brake cases and help to run the roads efficiently. The prototype serves as an impetus to drive future research, geared towards developing a more robust and embedded real-time fingerprint based licensing systems in vehicles. 


\section{ACKNOWLEDGMENTS}

Our thanks to the experts from PICT Pune who have contributed towards design of the system.

\section{REFERENCES}

[1] S. Ashwin, S. Loganathan, S. Santosh Kumar, P. Sivakumar, "Prototype of a fingerprint based licensing system for driving",2013 International Conference on Information Communication and Embedded Systems (ICICES), Year 2013.

[2] Rubella, J. Angeline, et al. "Fingerprint based license checking for auto-mobiles."Advanced Computing (ICoAC), 2012 Fourth International Conference on. IEEE, 2012.

[3] David Silcock, Anna Sunter \& Chris van Lottum, Ross
Silcock Limited, Kris Beuret, Social Research Associates, "Unlicenced Driving: A Scoping Study to Identify Potential Areas for Further Research" Foundation for Road Safety Research.

[4] Anil Jain, Arun Ross and Salil Prabhakar, "Fingerprint Matching Using Minutiae and Texture Features," Fingerprint Matching Using Minutiae and Texture Features", in Proc. of Int'l Conference on Image Processing (ICIP), pp.282-285, Thessaloniki, Greece Oct 7 - 10, 2001

[5] https://en.wikipedia.org/wiki/Regional_Transport_Office

[6] https://www.arduino.cc/

[7] https://www.raspberrypi.org 